\title{
A randomised trial comparing laparoscopy with laparotomy in the management of women with ruptured ectopic pregnancy
}

\author{
L C Snyman, BMedSci, MB ChB, MPraxMed, MMed (O\&G), FCOG (SA); T Makulana, MB ChB, MMed (O\&G), FCOG (SA); \\ J D Makin, MB ChB, BSc Hons (Epi)
}

Department of Obstetrics and Gynaecology, Faculty of Health Sciences, School of Medicine, University of Pretoria and Kalafong Provincial Tertiary Hospital, Pretoria, South Africa

Corresponding author: L C Snyman (leon.snyman@up.ac.za)

\begin{abstract}
Background. Ruptured ectopic pregnancy (REP) is a common gynaecological emergency in resource-poor settings, where laparotomy is the standard treatment despite laparoscopic surgery being regarded as the optimal treatment. There is a lack of prospective randomised data comparing laparoscopic surgery with laparotomy in the surgical management of women with REP.

Objective. To compare operative laparoscopy with laparotomy in women with REP.

Methods. This was a randomised parallel study. One hundred and forty women with suspected REP were randomised to undergo operative laparoscopy or laparotomy. The outcome measures were operating time, hospital stay, pain scores and analgesic requirements, blood transfusion, time to return to work, and time to full recovery.

Results. Operating time was significantly longer in the laparoscopy group (67.3 v. 30.5 minutes, $p<0.001)$. Duration of hospital stay, pain scores and need for analgesia were significantly less in the laparoscopy group. Women in this group returned to work 8 days earlier and their time to full recovery was significantly shorter compared with those in the laparotomy group. Significantly more women undergoing laparotomy required blood transfusion than women in the laparoscopy group. In the latter group, $14.5 \%$ of women required blood transfusion compared with $26.5 \%$ in the laparotomy group $(p=0.01)$.

Conclusion. Operative laparoscopy in women treated for REP is feasible in a resource-poor setting and is associated with significantly less morbidity and a quicker return to economic activity.
\end{abstract}

S Afr Med J 2017;107(3):258-263. DOI:10.7196/SAMJ.2017.v107i3.11447

Ectopic pregnancies are common gynaecological emergencies managed in our institution. The majority of women with this condition present with ruptured or bleeding tubal pregnancies, mainly due to poor contraception services, high numbers of unintended pregnancies, and few early pregnancy confirmation visits. The current standard of treatment at our institution, as in most other public hospitals in South Africa (SA) and elsewhere in resource-poor settings, is emergency laparotomy with salpingectomy of the involved fallopian tube. ${ }^{[1-4]}$ At our institution we offer women with suspected unruptured tubal pregnancies laparoscopic surgical treatment.

There is a lack of prospective randomised data with regard to the optimal surgical treatment of women with ruptured ectopic pregnancies (REPs). According to a meta-analysis of two studies involving haemodynamically stable women with small, unruptured tubal ectopic pregnancies, laparoscopic salpingostomy was significantly inferior to salpingostomy by laparotomy for successfully treating tubal ectopic pregnancy. ${ }^{[5-7]}$ However, the laparoscopic approach was superior to laparotomy in terms of significantly shorter operating time, ${ }^{[6,7]}$ reduced intraoperative blood loss, analgesic requirement, time to normal activity, total hospital costs, ${ }^{[8]}$ shorter hospital stay and quicker recovery in haemodynamically stable women. ${ }^{[9]}$

Although some data suggest that laparoscopic surgery is feasible in women with significant haemoperitoneum, ${ }^{[10-13]}$ to our knowledge there are no prospective randomised trials comparing laparotomy with laparoscopy in the surgical management of women with REP in any setting. The available evidence of the preferred mode of surgical treatment for women diagnosed with suspected REP is scanty and consists of retrospective trials, mostly with small numbers. ${ }^{[14]}$ REPs were excluded from the two trials analysed in the Cochrane metaanalysis on this topic. ${ }^{[6,7]}$

Decision-making with regard to surgical treatment of women with REP in resource-poor settings is important and has significant consequences. If laparoscopic management is beneficial and feasible, it requires a substantial investment in equipment, instruments and skills training to enable as many women as possible to benefit from minimally invasive procedures. If, however, there are no differences in outcomes between the two surgical modalities, the status quo could be maintained, with the reassuring knowledge that women are not being deprived of a better alternative surgical approach.

The objective of this study was to assess the feasibility of laparoscopic surgery in the management of women diagnosed with suspected REP at Kalafong Provincial Tertiary Hospital (KPTH), Pretoria, SA and to compare laparoscopic surgery with laparotomy with regard to certain outcomes.

\section{Methods}

This was a randomised parallel trial conducted at KPTH between May 2012 and November 2013. KPTH accepts referrals and provides all levels of gynaecological care to women in the South Western District of the Tshwane Metropolitan area. It is also part of the training circuit for registrars in the Department of Obstetrics and Gynaecology, University of Pretoria. 
Eligible participants were women $\geq 18$ years of age who were to undergo emergency surgical treatment following a clinical diagnosis of REP. Women who were willing and able to provide informed consent and met the inclusion criteria were recruited to the trial.

As laparotomy is the standard treatment at our institution and the registrars and consultants performing laparoscopic surgery were all trained but inexperienced laparoscopic surgeons, two of three parameters (haemoglobin value $\geq 8 \mathrm{~g} / \mathrm{dL}$, systolic blood pressure $\geq 90 \mathrm{mmHg}$, and pulse rate $\leq 100$ beats/minute) had to be met for women to be included in the trial. Women who did not meet the inclusion criteria underwent standard treatment.

Women recruited to the study were randomised to undergo either laparotomy and salpingectomy (standard treatment) or laparoscopic salpingectomy of the involved fallopian tube. Simple randomisation was done after recruitment by means of sequentially numbered, opaque, sealed envelopes. Seventy labels with the word 'laparotomy' and another 70 labels with the word 'laparoscopy' were inserted in opaque (when held to light) envelopes and sealed. The envelopes were shuffled and then numbered from 1 to 140 . Recruited participants were sequentially numbered and the appropriate envelope was opened only after the participant's information was recorded in the research file. The registrar on call for gynaecology did recruitment and randomisation of participants.

Salpingectomy of the affected fallopian tube through laparotomy was performed through a midline or transverse incision according to the treating physician's preference. Laparoscopic procedures were performed using 3 or 4 re-usable or disposable ports $(2 \times 10 \mathrm{~mm}$ and $2 \times 5 \mathrm{~mm}$ ) placed at the treating physician's discretion. Suction was provided using a $5 \mathrm{~mm}$ or $10 \mathrm{~mm}$ re-usable suction device. Salpingectomy was done using re-usable bipolar coagulation (Roby device, Karl Storz Endoskope, Germany) and disposable laparoscopic scissors. In cases where technical difficulties were experienced and laparoscopy was consequently not possible as a treatment option, conversion to laparotomy was allowed. Rotating registrars and consultants in the department performed all procedures.

Postoperative care was as per routine in the gynaecology ward and the same for both groups. Opioid analgesia for the first 24 hours postoperatively was prescribed in combination with paracetamol and non-steroidal anti-inflammatory drugs (NSAIDs). On discharge, all women were offered 4 weeks' sick leave and received prescriptions for paracetamol and NSAIDs for $7-10$ days. Arrangements for removal of the sutures at the local clinic were made for 7 days after the procedure.

Assessment of postoperative pain was done using a Wong-Baker FACES Pain Rating Scale (USA), with 6 facial expressions suggesting various pain intensities and a score out of 10 once during 24 hours after the procedure.

Telephonic follow-up was conducted for all the patients 2 weeks after discharge to determine when they resumed normal activity, if they were still administering pain medication, or had any other postoperative complaints or problems. The telephonic interview was conducted by an interviewer blinded for the type of procedure the participant had undergone. Some participants had to be interviewed more than once.

The outcomes were duration of hospital stay, number of days before participants returned to full employment, pain scores, pre- and postoperative haemoglobin values, need for blood transfusion, and operating time.

For sample size calculations the $\alpha$-value was set at 0.05 and the $\beta$-value at 0.02 . The outcome variables were 4 days' hospital stay and 4 weeks to return to full employment following laparotomy for REP. The required sample size to detect the minimal clinically significant differences expected between the laparotomy and laparoscopy groups for both outcomes was 63 women in each arm. To compensate for possible loss of follow-up of $\sim 10 \%$, it was decided to recruit 140 women to the study.

Analysis was performed on an intention-to-treat basis. Data were entered into a Microsoft Excel (USA) spreadsheet. Duplicates were removed and missing data were added, if available. Outliers were investigated and adjusted if found to be incorrect. The data were imported into SPSS Version 21 (IBM Corp., USA) for analysis. Data analysis was done by descriptive statistics to describe means and standard deviations in cases of continuous data and frequencies. Continuous normally distributed data were analysed using Student $t$-tests for independent samples. Continuous data that were not normally distributed, e.g. operating time, were analysed making use of the Mann-Whitney $U$-test (non-parametric). $\chi^{2}$ or Fischer exact tests (where appropriate) were used in the case of categorical data.

Operating time is a function of the type of intervention and type of operator. Multinomial logistic regression was used, with operator and intervention entered into the model as the independent variables, thus controlling for the effect of the operator. Consultants and registrars performed all laparoscopy and laparotomy procedures, while rotating interns performed some of the laparotomy procedures, as this is part of their training requirements.

There were 15 women randomised to undergo laparoscopic procedures in whom laparotomies were performed. A sub-analysis was done to assess the effect of the actual intervention received. This was done as per the initial analysis.

The study was approved by the Health Sciences Research Ethics Committee, University of Pretoria (ref. no. 244/2011) and was registered as a clinical trial with ClinicalTrials.gov (clinical trials ID: NCT01932957)

\section{Results}

During the study period, 410 women with ectopic pregnancies were eligible for recruitment to the trial. One hundred and forty women with REPs were randomised into the two treatment arms (70 women in each group). Data of 139 women were available for analysis. One

Table 1. Demographic data and preoperative haemoglobin values

\begin{tabular}{lllll}
\hline Measure & Laparoscopy $(\boldsymbol{N = 7 0})$ & Patients, $\boldsymbol{n}$ & Laparotomy $(\boldsymbol{N}=\mathbf{6 9})$ & Patients, $\boldsymbol{n}$ \\
\hline Age (years), mean (SD) & $28.2(5.8)$ & 70 & $28.3(6.0)$ & 69 \\
Parity, median (IQR) & $1(2)$ & 70 & $1(2)$ & 68 \\
Gravidity, median (IQR) & $2(2)$ & 70 & $3(1)$ & 68 \\
Previous surgery, frequency (\%) & $7(10.6)$ & 66 & $10.8(2.0)$ & 66 \\
Preoperative Hb, mean (SD) & $11.1(1.6)$ & 69 & & 69 \\
SD = standard deviation; IQR = interquartile range. & & & &
\end{tabular}


woman randomised to the trial presented with a second ectopic pregnancy and was recruited and randomised for a second time. Her data for the second randomisation were excluded from the analysis in the laparotomy group. The flow diagram in Fig. 1 shows the reasons why women were not recruited to the trial.
Demographic data and preoperative haemoglobin values of the two groups were similar, as shown in Table 1, and there were no statistically significant differences between the two groups.

The mean operating time for laparoscopic procedures was 67.3 (standard deviation (SD) 51.1) minutes, and 30.5 (SD 13.9) minutes for proce-

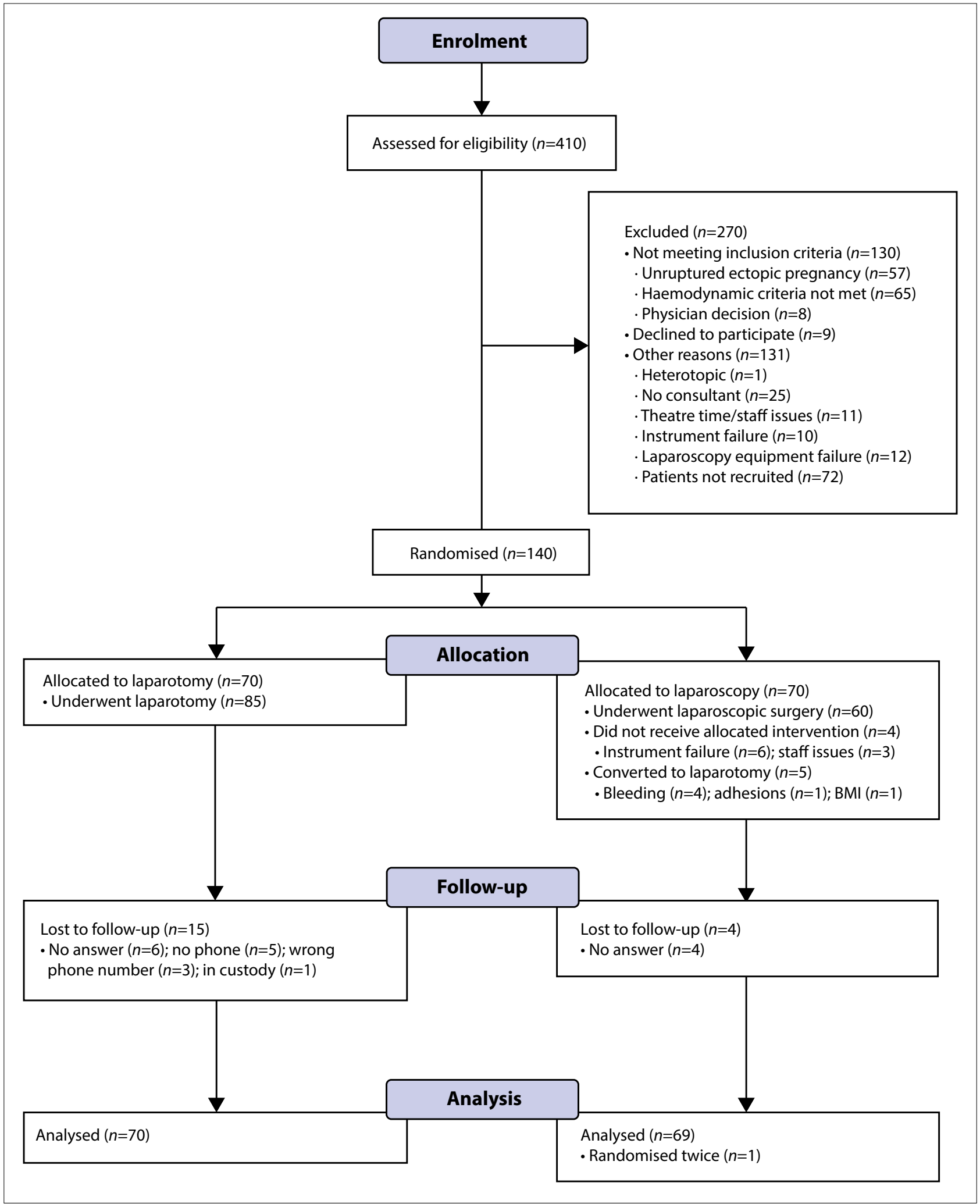

Fig. 1. Reasons why women with REP were not recruited to the trial. (BMI = body mass index.) 
dures performed by laparotomy $(p<0.0001)$. Hospital stay and pain scores were statistically significantly shorter and lower in the laparoscopy and laparotomy groups. Eighteen women (26.5\%) in the laparotomy group required blood transfusion compared with $10(14.5 \%)$ in the laparoscopy group $(p=0.01)$. The postoperative haemoglobin values were similar for the two groups (Table 2).

Telephonic follow-up was completed in the majority of patients. There was no statistically significant difference in the two groups with regard to the number of days lapsed between discharge and telephonic follow-up.

Nineteen women were lost to follow-up. Of these, 15 (22\%) were in the laparotomy group and $4(5.7 \%)$ in the laparoscopy group. Reasons for lost-to-follow-up included no answer $(n=10)$, no phone $(n=5)$, incorrect cellular phone number $(n=3)$, and prisoner in custody $(n=1)$.

Women in the laparoscopy group reported experiencing no pain after a mean (SD) of 8.1 (8.3) days compared with a mean (SD) of 13.26 (1.3) days for women in the laparotomy group $(p<0.0001)$. Women in the laparoscopy group also reported a statistically significant shorter period from operation to normal function than those in the laparotomy group (Table 2).

Fifteen women $(21.4 \%)$ who were randomised to undergo laparoscopic procedures underwent laparotomy. Instrument failure occurred in 6 women, and there were staff-related issues in another 4 cases. Laparoscopic procedures were converted to laparotomy because of bleeding in 4 cases, and adhesions and obesity in 1 case each.
The results of a sub-analysis of actual intervention are shown in Table 3. In this analysis, operating times remained longer in the laparoscopy group, but the differences between the two groups were slightly less (24.25 and 36.8 minutes, respectively). Hospital stay and pain scores remained statistically significantly shorter in the laparoscopy group, and more women in the laparoscopy group did not require blood transfusion. The mean number of days to normal functioning in the laparoscopy group was even shorter - at 6.5 days in the actual intervention group and 8.2 days in the intention-to-treat group.

Multinomial logistic regression was used, with operator and intervention entered into the model as independent variables, thus controlling for the effect of the operator. The results are shown in Table 4 .

\section{Discussion}

This article presents the results of a randomised prospective trial comparing laparotomy with laparoscopy in the surgical management of women with REP. Provision had to be made to exclude women from recruitment to the study where there were concerns with regard to their haemodynamic status. This was done to ensure patient safety, as the standard of care in the institution is laparotomy, and emergency and after-hours laparoscopic surgery for this group of women is a relatively unfamiliar concept in the hospital setting. Consequently, there was a learning curve involved for surgeons, anaesthetists and theatre nursing staff. However, there is substantial evidence in the published literature suggesting that it

Table 2. Postoperative outcomes

\begin{tabular}{|c|c|c|c|c|c|}
\hline Measure & $\begin{array}{l}\text { Laparoscopy } \\
(N=70)\end{array}$ & Patients, $n$ & $\begin{array}{l}\text { Laparotomy } \\
(N=69)\end{array}$ & Patients, $n$ & $p$-value \\
\hline Operating time, $\min (\%)$ & $67.3(51.1)$ & 69 & $30.5(13.9)$ & 68 & $<0.0001$ \\
\hline Median (IQR) & $55(54)$ & & $30(15)$ & & \\
\hline \multicolumn{6}{|l|}{ Categorised operating time, $\min (\%)$} \\
\hline$<30$ & $8(11.4)$ & & $30(44.1)$ & & $<0.0001$ \\
\hline $30-59$ & $31(44.3)$ & & $34(50)$ & & \\
\hline$\geq 60$ & $31(44.3)$ & & $4(5.9)$ & & \\
\hline \multicolumn{6}{|l|}{ Hospital stay (days) } \\
\hline Mean (SD) & $1.7(1.0)$ & 66 & $3.0(0.8)$ & 63 & $<0.0001$ \\
\hline Median (IQR) & $1(2)$ & & $3(0)$ & & \\
\hline Pain score & $3.5(2.1)$ & 69 & $4.9(2.1)$ & 69 & $<0.0001$ \\
\hline \multicolumn{6}{|l|}{ Blood transfused, $n(\%)$} \\
\hline No transfusion & $59(85.5)$ & 69 & $50(73.5)$ & 67 & 0.01 \\
\hline $1-2 U$ & $7(10.1)$ & & $18(26.5)$ & & \\
\hline $3-4 \mathrm{U}$ & $3(4.4)$ & & $0(0)$ & & \\
\hline Postoperative $\mathrm{Hb}$ & $10.4(1.6)$ & 68 & $9.9(1.8)$ & 65 & NS \\
\hline \multicolumn{6}{|l|}{ Time to no pain and recovery } \\
\hline Duration to no pain, days (\%) & $8.1(8.3)$ & 66 & $13.26(1.3)$ & 53 & $<0.0001$ \\
\hline Median (IQR) & $4(7)$ & & $14(7)$ & & \\
\hline$<7$ & $38(56.7)$ & & $7(13.2)$ & & $<0.0001$ \\
\hline $7-13$ & $11(16.4)$ & & $11(20.8)$ & & \\
\hline $14-20$ & $7(10.4)$ & & $20(37.7)$ & & \\
\hline$\geq 21$ & $11(16.4)$ & & $15(28.3)$ & & \\
\hline Duration to normal functioning, days (\%) & $8.2(7.0)$ & 66 & $14.3(7.5)$ & 54 & $<0.0001$ \\
\hline$<7$ & $36(54.5)$ & & $6(11.1)$ & & $<0.0001$ \\
\hline $7-13$ & $10(15.2)$ & & $12(22.2)$ & & \\
\hline $14-20$ & $12(18.2)$ & & $22(40.7)$ & & \\
\hline$\geq 21$ & $8(12.1)$ & & $14(25.9)$ & & \\
\hline
\end{tabular}




\begin{tabular}{|c|c|c|c|c|c|}
\hline Measure & $\begin{array}{l}\text { Laparoscopy } \\
(N=70)\end{array}$ & Patients, $n$ & $\begin{array}{l}\text { Laparotomy } \\
(N=69)\end{array}$ & Patients, $n$ & $p$-value \\
\hline Operating time (min), median (IQR) & $63.36(40.4)$ & 55 & $39.11(39.7)$ & 83 & $<0.0001$ \\
\hline \multicolumn{6}{|l|}{ Categorised operating time, $\min (\%)$} \\
\hline$<30$ & $5(9.1)$ & 55 & $33(39.8)$ & 83 & $<0.0001$ \\
\hline $30-59$ & $26(47.3)$ & & $39(47)$ & & \\
\hline$\geq 60$ & $24(43.6)$ & & $11(13.3)$ & & \\
\hline Hospital stay (days), mean (SD) & $1.38(0.81)$ & 53 & $3.01(0.70)$ & 77 & $<0.0001$ \\
\hline Pain score & $2.78(1.5)$ & 55 & $5.1(2.0)$ & 83 & $<0.0001$ \\
\hline \multicolumn{6}{|l|}{ Blood transfused, $n(\%)$} \\
\hline No transfusion & $50(90.9)$ & 55 & $59(72.0)$ & 82 & 0.015 \\
\hline $1-2 U$ & $5(9.1)$ & & $20(24.3)$ & & \\
\hline $3-4 \mathrm{U}$ & $0(0)$ & & $3(3.7)$ & & \\
\hline \multicolumn{6}{|l|}{ Duration to no pain, days (\%) } \\
\hline$<7$ & $37(69.8)$ & 53 & $8(11.9)$ & 67 & $<0.0001$ \\
\hline $7-13$ & $9(17)$ & & $13(19.4)$ & & \\
\hline $14-20$ & $4(7.5)$ & & $23(34.3)$ & & \\
\hline$\geq 21$ & $3(5.7)$ & & $23(34.3)$ & & \\
\hline Duration to normal functioning, days (\%) & $6.55(5.99)$ & 53 & $14.51(7.31)$ & 67 & \\
\hline$<7$ & $34(64.2)$ & & $8(11.9)$ & & $<0.0001$ \\
\hline $7-13$ & $10(18.9)$ & & $12(17.9)$ & & \\
\hline $14-20$ & $5(9.4)$ & & $27(40.3)$ & & $<0.0001$ \\
\hline$\geq 21$ & $4(7.5)$ & & $20(29.9)$ & & \\
\hline
\end{tabular}

Table 4. Multinomial logistic regression for laparoscopy and laparotomy procedures

\begin{tabular}{|c|c|c|c|c|c|c|c|}
\hline \multirow{2}{*}{$\begin{array}{l}\text { Measure } \\
\text { Operating time }(\min )\end{array}$} & \multicolumn{3}{|c|}{ Laparoscopy, frequency (\%) } & \multicolumn{4}{|c|}{ Laparotomy, frequency (\%) } \\
\hline & Consultant & Registrar & $p$-value & Consultant & Registrar & Intern & $p$-value \\
\hline$<30$ & $2(6.1)$ & $6(18.2)$ & & $4(80.0)$ & $21(40.4)$ & $5(41.7)$ & \\
\hline $30-59$ & $12(36.4)$ & $18(51.5)$ & & $0(0.0)$ & $29(55.8)$ & $6(50.0)$ & \\
\hline$\geq 60$ & $19(57.6)$ & $12(30.3)$ & 0.15 & $1(20.0)$ & $2(3.8)$ & $1(8.3)$ & 0.15 \\
\hline
\end{tabular}

is safe and feasible to perform laparoscopic procedures for women with REP in the presence of significant haemoperitoneum or even hypovolaemic shock. ${ }^{[10-13]}$

Regression analysis accounting for the learning curve shows that laparoscopic surgery requires longer operating time; this is a feature of the procedure and not exclusively operator dependent. In this study, operating time was significantly longer for laparoscopic surgery compared with laparotomy. This finding is in contrast with that in other studies reporting on management of ectopic pregnancies. In the trials published by Lundorff et al. ${ }^{[6]}$ and Murphy et al. ${ }^{[8]}$ randomisation was done after confirmation of the diagnosis of unruptured ectopic pregnancy after diagnostic laparoscopy, but time was measured from the onset of the diagnostic laparoscopy until completion of the surgery. Randomised controlled trials reporting outcomes of laparoscopic surgery in other gynaecological procedures have also shown longer operating times associated with laparoscopic surgery. ${ }^{[14-17]}$ In $55 \%$ of women, operating time for the laparoscopic procedure was $\leq 60$ minutes. The increase in operating time is to a large extent compensated for by the shorter hospital stay associated with laparoscopy.

The data from this trial confirm the well-recognised benefits of laparoscopic surgery, such as shorter hospital stay, less pain and quicker recovery, which are also applicable to young healthy women.
Pain scores and need for analgesia were significantly lower after laparoscopic surgery. Hospital stay was significantly shorter in the laparoscopy group than in the laparotomy group.

The recovery period in the laparoscopy group was significantly shorter. The majority of women in this group regarded themselves as fully recovered within 7 days from the day of the operation compared with $<50.0 \%$ in the laparotomy group, where the majority regarded themselves as fully recovered after 14 days. The vast majority of women in the laparoscopic group were able to resume their normal duties 7 days earlier than those in the laparotomy group. This is a significant finding, as $54.2 \%$ of women who presented to KPTH during the study period were not formally employed and therefore did not have benefits, such as paid sick leave. Many of these women perform casual work and for them it is a case of no work, no pay; they rely heavily on their physical wellbeing for part-time or casual employment. The time needed for them to recover is crucial, because it has a significant financial impact.

There were no differences between the preoperative haemoglobin levels between the two groups. An important finding of this study is that statistically significantly fewer women in the laparoscopy group required blood transfusions than in the laparotomy group. These blood transfusions were appropriate, as reflected in the postoperative 
haemoglobin values of the two groups, which showed no significant difference. It is possible that laparotomy causes additional blood loss, which is an important finding, as blood products remain a scarce commodity and administering blood is not without risks.

\section{Conclusion}

The findings of this study confirm that laparoscopic surgery is superior to laparotomy in the surgical management of women with REP, resulting in lower pain scores, fewer blood transfusions, shorter hospital stay and fewer days needed for convalescence allowing women to return to work sooner at the cost of longer operating times. Dedicated skills training and continuous efforts in promoting laparoscopic surgery are required to ensure that the majority of women with REP are treated laparoscopically, regardless of haemodynamic stability. ${ }^{[18]}$ The benefits to women are so overwhelming that these measures should be implemented. Although haemodynamically unstable patients were excluded from this study, there is enough evidence to suggest that laparoscopic surgery can be safely performed in these women. ${ }^{[11,12,19]}$

Secondary and tertiary public hospitals should invest in skills training, laparoscopic equipment and instruments to enable laparoscopic surgery for women presenting with ectopic pregnancy. Operative laparoscopy on a 24-hour basis for the treatment of women with REP is feasible in a public hospital in resource-poor settings and should become standard treatment for the majority of women with this condition.

1. Akaba GO, Agida TE, Onafowokan O. Ectopic pregnancy in Nigeriảs federal capital territory: A six year review. Niger J Med 2012;21(2):241-245.

2. Swende TZ, Jogo AA. Ruptured tubal pregnancy in Makurdi, north central Nigeria. Niger J Med 2008;17(1):75-77. http://dx.doi.org/10.4314/njm.v17i1.37361

Obeidat B, Zayed F, Amarin Z, Obeidat N, El-Jallad MF. Tubal ectopic pregnancy in the north of Jordan: Presentation and management. Clin Exp Obstet Gynecol 2010;37(2):138-140.

4. Goyaux N, Leke R, Keita N, Thonneau P. Ectopic pregnancy in African developing countries. Acta Obstet Gynecol Scand 2003:82(4):305-312. http://dx doi.org/10.1034/j.1600-0412.2003.00175
5. Hajenius PJ, Mol F, Mol BW, Bossuyt PM, Ankum WM, van der Veen F. Interventions for tubal ectopic pregnancy. Cochrane Database Syst Rev 2007;(1):CD000324. http://dx.doi.org/10.1002/14651858. CD000324. pub2

6. Lundorff $\mathrm{P}$, Thorburn J, Hahlin M, Kallfelt B, Lindblom B. Laparoscopic surgery in ectopic pregnancy. A randomized trial versus laparotomy. Acta Obstet Gynecol Scand 1991;70(4-5):343-348. http://dx.doi. org $/ 10.3109 / 00016349109007885$

7. Vermesh M, Silva PD, Rosen GF, Stein AL, Fossum GT, Sauer MV. Management of unruptured ectopic gestation by linear salpingostomy: A prospective, randomized clinical trial of laparoscopy versus laparotomy. Obstet Gynecol 1989,73(3)

8. Murphy AA, Nager CW, Wujek JJ, Kettel LM, Torp VA, Chin HG. Operative laparoscopy versus laparotomy for the management of ectopic pregnancy: A prospective trial. Fertil Steril 1992;57(6):11801185. http://dx.doi.org/10.1016/s0015-0282(16)55070-5

9. Saleh AM, Mahjoub MM, El-Kurdy AM. Laparoscopy versus laparotomy management of tubal pregnancy. Saudi Med J 2001;22(9):771-775. http://dx.doi.org/10.1016/j.ijgo.2013.05.014

10. Cohen A, Almog B, Satel A, Lessing JB, Tsafrir Z, Levin I. Laparoscopy versus laparotomy in the management of ectopic pregnancy with massive hemoperitoneum. Int I Gynaecol Obstet 2013;123(2):139-141. http:///dx.doi.org/10.1016/.ijgo.2013.05.014

11. Rizzuto MI, Oliver R, Odejinmi F. Laparoscopic management of ectopic pregnancy in the presence of a significant haemoperitoneum. Arch Gynecol Obstet 2008;277(5):433-436. http://dx.doi.org/10.1007/ s00404-007-0473-7

12. Takeda A, Manabe S, Mitsui T, Nakamura H. Management of patients with ectopic pregnancy with massive hemoperitoneum by laparoscopic surgery with intraoperative autologous blood transfusion. J Minim Invasive Gynecol 2006;13(1):43-48. http://dx.doi.org/10.1016/j.jmig.2005.09.100

13. Tulandi T, Kabli N. Laparoscopy in patients with bleeding ectopic pregnancy. J Obstet Gynaecol Can 2006;28(5):361-365. http://dx.doi.org/10.1016/s1701-2163(16)32157-0

14. Lo L, Pun TC, Chan S. Tubal ectopic pregnancy: An evaluation of laparoscopic surgery versus laparotomy in 614 patients. Aust N Z J Obstet Gynaecol 1999;39(2):185-187. http://dx.doi.org/10.1111/ j.1479-828x.1999.tb03368.x

15. Medeiros LR, Stein AT, Fachel J, Garry R, Furness S. Laparoscopy versus laparotomy for benign ovarian tumor: A systematic review and meta-analysis. Int J Gynecol Cancer 2008;18(3):387-399. http://dx.doi, org/10.1111/j.1525-1438.2007.01045.x

16. Nieboer TE, Johnson N, Lethaby A, et al. Surgical approach to hysterectomy for benign gynaecological disease Cochrane Database Syst Rev 2009:(2):CD003677 http///dx doi.org/10.1002/14651858. CD003677.pub4

17. Zullo F, Falbo A, Palomba S. Safety of laparoscopy vs laparotomy in the surgical staging of endometrial Zullo F, Falbo A, Palomba S. Safety of laparoscopy vs laparotomy in the surgical staging of endometrial
cancer: A systematic review and metaanalysis of randomized controlled trials. Am I Obstet Gynecol cancer: A systematic review and metaanalysis of randomized 18. Odejinmi F, Rizzuto I, Oliver R, Alalade A, Agarwal N, Olowu O. Beyond guidelines: Effectiveness of a
programme in achieving operative laparoscopy for all women requiring surgical management of ectopic pregnancy. Gynecol Obstet Invest 2015;80(1):46-53. http://dx.doi.org/10.1159/000371763

9. Cengiz H, Kaya C, Ekin M, Karakas S, Yasar L. Is laparoscopic surgery safe in patients with an elevated shock index due to ruptured ectopic pregnancy? Clin Exp Obstet Gynecol 2013;40(3):418-420.

Accepted 10 January 2017 